\title{
Triglyceride/low-density-lipoprotein cholesterol ratio is the most valuable predictor for increased small, dense LDL in type 2 diabetes patients
}

\author{
Gen Ouchi ${ }^{1 \dagger}$, Ichiro Komiya ${ }^{2,3^{*+}}$ (D), Shinichiro Taira ${ }^{4}$, Tamio Wakugami ${ }^{2}$ and Yusuke Ohya ${ }^{5}$
}

\begin{abstract}
Background: Small, dense low-density lipoprotein (sd-LDL) increases in type 2 diabetes patients and causes arteriosclerosis. Non-high-density-lipoprotein cholesterol (non-HDL-C) is thought to be useful for predicting arteriosclerosis and sd-LDL elevation; however, there are no data about whether the triglyceride /low-densitylipoprotein cholesterol (TG/LDL-C) ratio is a valuable predictor for sd-LDL.

Methods: A total of 110 type 2 diabetes patients with hypertriglyceridemia were analyzed. No patients were treated with fibrates, but 47 patients were treated with statins. LDL-C was measured by the direct method. LDLmigration index (LDL-MI) using electrophoresis (polyacrylamide gel, PAG) was calculated, and a value $\geq 0.400$ was determined to indicate an increase in sd-LDL. Simple regression analyses were carried out between LDL-MI and lipid markers. Receiver operating characteristic curves of lipid markers for predicting high LDL-MI were applied to determine the area under the curve (AUC), sensitivity, specificity, and cut-off point.

Results: LDL-MI correlated negatively with LDL-C $(P=0.0027)$ and PAG LDL fraction $(P<0.0001)$ and correlated positively with TGs, non-HDL-C, TG/LDL-C ratio, TG/HDL-C ratio, and non-HDL-C/HDL-C ratio among all study patients. Similar results were obtained for patients analyzed according to statin treatment. The AUCs (95\% confidence interval) were 0.945 (0.884-1.000) for TG/LDL-C ratio and $0.614(0.463-0.765)$ for non-HDL-C in patients without statins $(P=0.0002)$. The AUCs were 0.697 (0.507-0.887) for TG/LDL-C and 0.682 (0.500-0.863) for non-HDL-C in patients treated with statins. The optimal cut-off point for TG/LDL-C ratio for increased LDL-MI was 1.1 (molar ratio) regardless of statin treatment. The sensitivity and specificity of the TG/LDL-C ratio $(90.0$ and $93.9 \%$, respectively) were higher than those of non-HDL-C (56.7 and 78.8\%, respectively) in patients without statins.
\end{abstract}

Conclusions: The TG/LDL-C ratio is a reliable surrogate lipid marker of sd-LDL and superior to non-HDL-C in type 2 diabetes patients not treated with statins.

Keywords: TG/LDL-C ratio, Non-HDL-C, Small, Dense LDL, Triglycerides, Type 2 diabetes mellitus

\footnotetext{
* Correspondence: i.kom.iya@nifty.com

${ }^{\dagger}$ Gen Ouchi and Ichiro Komiya contributed equally to this work.

2Department of Internal Medicine, Okinawa Medical Hospital, 2310

Tsuhako-Nishihara, Sashiki, Nanjo, Okinawa 901-1414, Japan

${ }^{3}$ Department of Diabetes and Endocrinology, Medical Plaza Daido Central,

123 Daido, Naha, Okinawa 902-0066, Japan

Full list of author information is available at the end of the article
}

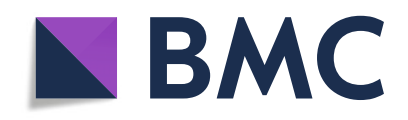

(- The Author(s). 2021 Open Access This article is licensed under a Creative Commons Attribution 4.0 International License, which permits use, sharing, adaptation, distribution and reproduction in any medium or format, as long as you give appropriate credit to the original author(s) and the source, provide a link to the Creative Commons licence, and indicate if changes were made. The images or other third party material in this article are included in the article's Creative Commons licence, unless indicated otherwise in a credit line to the material. If material is not included in the article's Creative Commons licence and your intended use is not permitted by statutory regulation or exceeds the permitted use, you will need to obtain permission directly from the copyright holder. To view a copy of this licence, visit http://creativecommons.org/licenses/by/4.0/. The Creative Commons Public Domain Dedication waiver (http://creativecommons.org/publicdomain/zero/1.0/) applies to the data made available in this article, unless otherwise stated in a credit line to the data. 


\section{Introduction}

The risk of cardiovascular disease (CVD) is reportedly associated with an increase in small, dense low-density lipoprotein (sd-LDL) levels rather than large, buoyant LDL [1]. Sd-LDL reportedly exhibits several potentially atherogenic properties, such as reduced receptormediated clearance, prolonged retention in circulation, greater arterial wall retention, and increased oxidation [2]. Japanese elderly men with ischemic heart disease and high sd-LDL were shown to have increased risk of CVD events over the next 5 years [3]. Higher sd-LDL occurs when both non-high-density-lipoprotein cholesterol (non-HDL-C) and triglycerides (TGs) are high [4]. Non-HDL-C, having higher atherosclerosis-inducing properties than LDL-cholesterol (LDL-C), is increased in hypertriglyceridemia [5] and associated with increased sd-LDL [6]. According to current guidelines for arteriosclerosis, non-HDL-C should be evaluated instead of LDL-C in cases such as severe hypertriglyceridemia [7]. When the non-HDL-C level is high, cholesterollowering therapy is prioritized, as in LDL cholestrolemia $[7,8]$. When the TG level is $\geq 4.5 \mathrm{mmol} / \mathrm{L}$, Friedewald's formula cannot accurately evaluate LDL-C, so nonHDL-C is used as a marker instead of LDL-C.

A recent study evaluating the effect of pemafibrate on hypertriglyceridemia in type 2 diabetes reported that baseline LDL-C (measured via the direct method) and LDL fraction (polyacrylamide gel [PAG] electrophoresis) decreased with increasing baseline sd-LDL [9]. Pemafibrate, a selective peroxisome proliferatoractivated receptor alpha modulator, allows control of serum TG levels and sd-LDL, which were previously inadequate with conventional treatment $[10,11]$. In more than half of type 2 diabetes patients with hypertriglyceridemia, baseline LDL-C was relatively lower than after pemafibrate administration [9]. Higher baseline TG level also appears to be involved in the baseline sd-LDL increase and post-dose LDL-C increase. In untreated type 2 diabetes patients with hypertriglyceridemia, large, buoyant LDL was reduced, suggesting that it accounts for low serum LDL-C measurements.

The change in non-HDL-C may not accurately reflect the change in LDL composition; that is, if the increase in TGs and decrease in LDL (mainly large, buoyant LDL) occur synchronously, non-HDL-C does not change markedly. This was shown in a previous study examining the effect of pemafibrate [9]. Diabetes patients with high TG but normal or low LDL-C may have higher sd-LDL. It was hypothesized that the TG/LDL-C ratio would be useful for predicting higher sd-LDL or apolipoprotein-B (apoB)-containing lipoproteins as an alternative to nonHDL-C. The present study clarified whether the TG/ LDL-C ratio is more valuable than non-HDL-C or other lipid markers in predicting sd-LDL level in type 2 diabetes patients treated with or without statins.

\section{Materials and methods \\ Patients and study procedures}

Subjects in this retrospective study were outpatients with type 2 diabetes visiting the Medical Plaza Daido Central. A total of 130 patients with type 2 diabetes with hypertriglyceridemia were enrolled in this study after excluding heavy drinkers and patients with an estimated glomerular filtration rate $(\mathrm{eGFR})<45 \mathrm{~mL} / \mathrm{min}$. Five patients were excluded because PAG electrophoresis data were not available. Fifteen patients treated with conventional fibrates, ezetimibe, or sodium-glucose cotransporter 2 inhibitors were also excluded. The final study group consisted of 110 patients, including 47 patients treated with statins and 63 not treated with statins (Fig. 1). Because TG levels tend to fluctuate under the influence of diet [12], it was confirmed that fasting TGs were $\geq 1.7 \mathrm{mmol} / \mathrm{L}(150 \mathrm{mg} / \mathrm{dL})$ by repeated measurements in the 110 diabetes patients enrolled in the study. The average of 2 or 3 measurements was used as the baseline value. Blood samples were collected after 9-12 h of fasting. All 110 patients are still being followed, and more than half of them have been treated with fibrates. In this study, all baseline data were analyzed prior to intervention with fibrates.

Lipoprotein electrophoresis (PAG) was conducted in all patients. PAG electrophoresis revealed 4 lipoprotein fractions (HDL, LDL, midband, and very-low-density lipoprotein [VLDL]). The LDL-migration index (LDLMI) was calculated from the pattern of PAG electrophoresis according to a previous report $[13,14]$; that is, the PAG electrophoretic distance between the LDL and VLDL fractions was divided by that between the HDL and VLDL fractions. When this value was $\geq 0.400$, it was determined to indicate an increase in sd-LDL [13, 14]. LDL-C was measured via the direct homogenized method $[15,16]$. If the TG level was $11.3 \mathrm{mmol} / \mathrm{L}(1,000$ $\mathrm{mg} / \mathrm{dL}$ ) or higher, the LDL-C level measured by the direct method would be unreliable, but there were no such subjects.

\section{Statistical analysis}

Data are shown as the mean \pm SD (normal distribution), median (interquartile range [IQR]) (nonparametric distribution), or percentage. Results of TG and TG-related variable analyses are shown as the median (25-75\% quartiles, IQR) due to non-parametric distribution. Comparisons between groups were made using $t$-test, Mann-Whitney $U$ test, or the $\chi^{2}$ test. A simple regression analysis using the least squares method was applied for continuous variables (TGs, LDL-C, non - HDL-C, TG/LDL-C ratio, TG/HDL-C ratio, LDL/HDL-C ratio, 


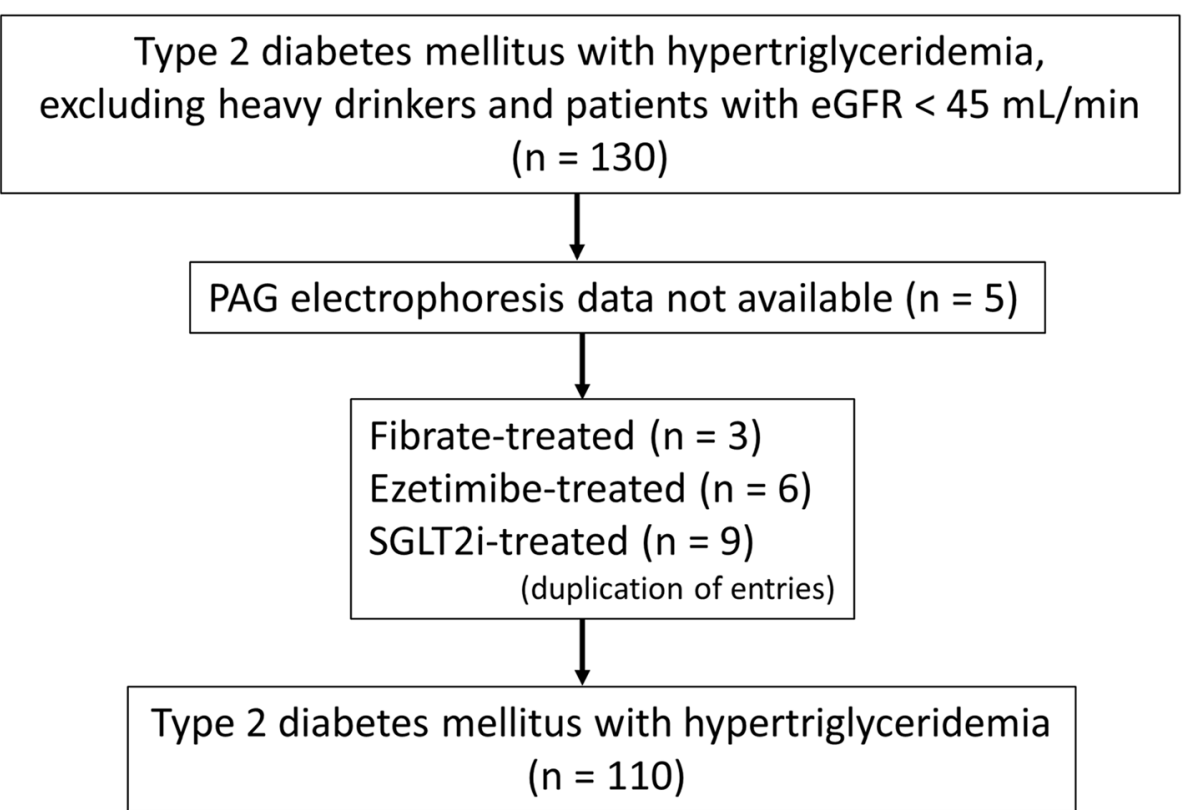

Fig. 1 Case-finding protocol. eGFR, estimated glomerular filtration rate; PAG, polyacrylamide gel; SGLT2i, sodium-glucose cotransporter 2 inhibitors

non-HDL-C/HDL-C ratio, LDL, midband, and VLDL fractions in PAG) as explanatory variables and with LDL-MI as a response variable to determine the regression coefficient $(r)$, standard error (SE), 95\% confidence interval $(\mathrm{CI})$, and standardized $R^{2}$ values. LDL-MI $\geq$ 0.400 was predictive of higher sd-LDL level. A receiver operating characteristic (ROC) curve was generated to evaluate the discriminatory ability of the variables for higher LDL-MI, and the area under the curve (AUC) with its 95\% CI was calculated. To determine the optimal cut-off points, sensitivity, and specificity of LDL-C, TGs, non-HDL-C, LDL and midband fractions in PAG, TG/LDL-C ratio, TG/HDL-C ratio, LDL-C/HDL-C ratio, and non-HDL-C/HDL-C ratio, the square root of ([1 sensitivity $^{2}+[1-\text { specificity }]^{2}$ ) was calculated, which represented the point on the ROC curve with the shortest distance from the upper left corner.

JMP for Windows software, version 12 (SAS Institute Japan; Tokyo, Japan), was used for statistical analyses. ROC analyses were performed using EZR (Saitama Medical Center, Jichi Medical University, Saitama, Japan), a graphical user interface for $\mathrm{R}$ (The $\mathrm{R}$ Foundation for Statistical Computing, Vienna, Austria) [17]. $P$ values of $<0.05$ were considered statistically significant.

\section{Results}

Comparison of clinical parameters in type 2 diabetes patients with hypertriglyceridemia

The clinical parameters of 110 type 2 diabetes patients are shown in Table 1 . The proportion of males was high. Mean body mass index was 26.7 (SD 3.9) $\mathrm{kg} / \mathrm{m}^{2}$. The average glycated hemoglobin was 7.3 $(1.4) \%$, and the average eGFR was $72.4(18.8) \mathrm{mL} /$ $\mathrm{min} / 1.73 \mathrm{~m}^{2}$. Hypertension and CVD/stroke complications were reported for 67.3 and $19.1 \%$ of patients, respectively. Comparing patients with and without statin treatment, the mean age was higher in patients treated with statins (66.6 [9.8] years) than in those not treated with statins (59.4 [13.6] years) $(P=0.0027$, $t$-test), and the average eGFR was lower in patients treated with statins $\left(66.6[13.4] \mathrm{mL} / \mathrm{min} / 1.73 \mathrm{~m}^{2}\right)$ than in those not treated with statins $(76.7[21.1] \mathrm{mL} / \mathrm{min} /$ $\left.1.73 \mathrm{~m}^{2}\right)(P=0.0051)$. However, there were no significant differences in other clinical parameters between the two groups. With regard to lipid markers, patients treated with statins were characterized by lower TGs, higher HDL-C, and lower non-HDL-C levels compared with patients not treated with statins. Patients treated with statins were characterized by lower TG/ HDL-C and non-HDL-C/HDL-C ratios compared with patients not treated with statins (Table 1).

Table 1 also shows the results of lipoprotein fraction analyses and LDL-MI in PAG electrophoresis. PAG electrophoresis revealed 4 lipoprotein fractions (HDL, LDL, midband, and VLDL), but there were cases in which the midband fraction was not present. The VLDL fraction was lower in patients treated with statins than in those not treated with statins (19.3 [SD 6.5]\% vs. $24.1[9.1] \%, P=0.0023)$. There was no statistically significant difference in LDL fraction 
Table 1 Characteristics of type 2 diabetes patients with hypertriglyceridemia

\begin{tabular}{|c|c|c|c|c|}
\hline Variables & Total & With statins & Without statins & $P^{*}$ \\
\hline Number (\%) & 110 & 47 & 63 & \\
\hline Age, mean $\pm S D$, years & $62.5 \pm 12.6$ & $66.6 \pm 9.8$ & $59.4 \pm 13.6$ & 0.0027 \\
\hline Men: Women & 70: 40 & $26: 21$ & 44: 19 & 0.1173 \\
\hline $\mathrm{BMI}$, mean $\pm \mathrm{SD}, \mathrm{kg} / \mathrm{m}^{2}$ & $26.7 \pm 3.9$ & $26.8 \pm 4.3$ & $26.6 \pm 3.6$ & 0.7968 \\
\hline $\mathrm{HbA} 1 \mathrm{c}$, mean $\pm \mathrm{SD}, \%$ & $7.3 \pm 1.4$ & $7.4 \pm 1.4$ & $7.2 \pm 1.5$ & 0.6683 \\
\hline $\mathrm{eGFR}$, mean $\pm \mathrm{SD}, \mathrm{mL} / \mathrm{min} / 1.73 \mathrm{~m}^{2}$ & $72.4 \pm 18.8$ & $66.6 \pm 13.4$ & $76.7 \pm 21.1$ & 0.0051 \\
\hline \multicolumn{5}{|l|}{ Complications } \\
\hline Hypertension, no. (\%) & $74(67.3)$ & $34(72.3)$ & $40(63.5)$ & 0.3279 \\
\hline CVD/stroke, no. (\%) & $21(19.1)$ & $9(19.2)$ & $12(19.1)$ & 0.9893 \\
\hline \multicolumn{5}{|l|}{ Treatment for diabetes } \\
\hline OHA, no. (\%) & $60(54.5)$ & $30(63.8)$ & $30(47.6)$ & 0.2835 \\
\hline Insulin + OHA, no. (\%) & $16(14.5)$ & $7(15.0)$ & $9(14.3)$ & \\
\hline Insulin, no (\%) & $4(3.6)$ & $1(2.1)$ & $3(4.8)$ & \\
\hline TG, median (IQR), mmol/L & $3.1(2.4-4.2)$ & $2.9(2.2-3.5)$ & $3.3(2.5-5.3)$ & 0.0167 \\
\hline $\mathrm{LDL}-\mathrm{C}$, mean $\pm \mathrm{SD}, \mathrm{mmol} / \mathrm{L}$ & $2.9 \pm 0.9$ & $2.9 \pm 0.7$ & $2.9 \pm 1.0$ & 0.9221 \\
\hline $\mathrm{HDL}-\mathrm{C}$, mean $\pm \mathrm{SD}, \mathrm{mmol} / \mathrm{L}$ & $1.2 \pm 0.3$ & $1.3 \pm 0.3$ & $1.2 \pm 0.3$ & 0.0150 \\
\hline Non $-\mathrm{HDL}-\mathrm{C}$, mean $\pm \mathrm{SD}, \mathrm{mmol} / \mathrm{L}$ & $4.6 \pm 1.0$ & $4.3 \pm 0.8$ & $4.8 \pm 1.1$ & 0.0122 \\
\hline TG/LDL-C ratio, median (IQR) & $1.0(0.8-1.7)$ & $0.9(0.7-1.3)$ & $1.1(0.8-2.2)$ & 0.0803 \\
\hline TG/HDL-C ratio, median (IQR) & $2.5(1.8-3.0)$ & $2.3(1.5-3.1)$ & $2.7(1.9-5.4)$ & 0.0169 \\
\hline LDL-C/HDL-C ratio, mean \pm SD & $2.5 \pm 0.9$ & $2.3 \pm 0.8$ & $2.6 \pm 0.9$ & 0.1050 \\
\hline Non $-\mathrm{HDL}-\mathrm{C} / \mathrm{HDL}-\mathrm{C}$ ratio, mean $\pm \mathrm{SD}$ & $4.1 \pm 1.8$ & $3.5 \pm 1.3$ & $4.5 \pm 2.0$ & 0.0039 \\
\hline \multicolumn{5}{|l|}{$\begin{array}{l}\text { Lipoprotein fraction } \\
\text { (PAG electrophoresis) }\end{array}$} \\
\hline $\mathrm{HDL}$, mean $\pm \mathrm{SD}, \%$ & $20.7 \pm 5.6$ & $21.4 \pm 4.5$ & $20.2 \pm 6.4$ & 0.2620 \\
\hline $\mathrm{LDL}$, mean $\pm \mathrm{SD}, \%$ & $39.7 \pm 12.7$ & $41.7 \pm 10.0$ & $38.2 \pm 14.3$ & 0.1544 \\
\hline Midband, mean $\pm S D, \%$ & $17.6 \pm 8.5$ & $17.7 \pm 7.8$ & $17.5 \pm 9.0$ & 0.9419 \\
\hline$V L D L$, mean $\pm S D, \%$ & $20.1 \pm 8.4$ & $19.1 \pm 6.5$ & $24.1 \pm 9.1$ & 0.0023 \\
\hline LDL-MI, median (IQR) & $0.393(0.353-0.420)$ & $0.381(0.350-0.404)$ & $0.398(0.355-0.426)$ & 0.0781 \\
\hline
\end{tabular}

${ }^{*} P$, t-test or Mann-Whitney $\mathrm{U}$ test between patients with and without statins

$B M I$ body mass index, HbA1c glycated hemoglobin, eGFR estimated glomerular filtration rate, CVD cardiovascular diseases, OHA oral hypoglycemic agents, TG triglycerides, $L D L-C$ low-density lipoprotein cholesterol, Non - HDL-C non - high-density lipoprotein cholesterol, $H D L-C$ high-density lipoprotein cholesterol, $L D L-M I$ LDL migration index, PAG polyacrylamide gel.

between patients treated with statins and those not treated with statins (41.7 [10.0]\% vs. $38.3[14.3] \%, P=$ $0.1544)$. The LDL-MI in patients treated with statins was 0.381 (IQR 0.350-0.404), which was lower than that in patients not treated with statins $(0.498$ [0.355$0.426])$, but the difference was not statistically significant $(P=0.0781)$.

\section{Simple regression analyses between lipid markers and LDL-MI}

In simple regression correlation analyses of the 110 patients, LDL-MI levels correlated with 10 lipid markers, except LDL-C/HDL-C ratio (Table 2). An inverse correlation was observed between LDL-MI and LDL fraction (PAG) $\left(R^{2}=0.2396, P<0.0001\right.$, least squares) and LDL-MI and LDL-C $\left(R^{2}=0.0804, P=0.0027\right)$. Positive correlations were observed between LDL-MI and the other 7 lipid markers. When patients were analyzed based on statin treatment status, an inverse correlation was observed between LDL-MI and LDL fraction in patients treated with statins $\left(R^{2}=0.1351, P=0.0113\right)$ and in those not treated with statins $\left(R^{2}=0.2668, P<\right.$ 0.0001). Results similar to those obtained for all study patients were observed when examining the relationship between the other 9 lipid markers and LDL-MI according to statin treatment status. 
Table 2 Simple regression analysis between LDL-MI and lipid markers in 110 type 2 diabetes patients with hypertriglyceridemia

\begin{tabular}{|c|c|c|c|c|c|c|}
\hline \multirow[t]{2}{*}{ Variables } & \multirow[t]{2}{*}{$\mathbf{r}$} & \multirow[t]{2}{*}{ SE } & \multicolumn{2}{|l|}{$95 \% \mathrm{Cl}$} & \multirow[t]{2}{*}{$P^{*}$} & \multirow{2}{*}{$\begin{array}{l}\text { Adjusted } \\
\mathrm{R}^{2}\end{array}$} \\
\hline & & & Lower & Upper & & \\
\hline \multicolumn{7}{|l|}{ Total } \\
\hline $\mathrm{TG}, \mathrm{mmol} / \mathrm{L}$ & 0.0188 & 0.0021 & 0.0147 & 0.0229 & $<0.0001$ & 0.4429 \\
\hline $\mathrm{LDL}-\mathrm{C}, \mathrm{mmol} / \mathrm{L}$ & -0.0183 & 0.0060 & -0.0301 & -0.0065 & 0.0027 & 0.0804 \\
\hline Non - HDL-C, mmol/L & 0.0207 & 0.0050 & 0.0108 & 0.0307 & $<0.0001$ & 0.1359 \\
\hline $\mathrm{TG} / \mathrm{LDL}-\mathrm{C}$ ratio & 0.0222 & 0.0030 & 0.0161 & 0.0282 & $<0.0001$ & 0.3299 \\
\hline TG/HDL-C ratio & 0.0109 & 0.0015 & 0.0080 & 0.0139 & $<0.0001$ & 0.3390 \\
\hline LDL-C/HDL-C ratio & 0.0019 & 0.0063 & -0.0105 & 0.0144 & 0.7572 & 0.0009 \\
\hline Non - HDL-C/HDL-C ratio & 0.0150 & 0.0027 & 0.0097 & 0.0203 & $<0.0001$ & 0.2252 \\
\hline LDL (PAG), \% & -0.0021 & 0.0004 & -0.0029 & -0.0014 & $<0.0001$ & 0.2396 \\
\hline Midband (PAG), \% & 0.0031 & 0.0006 & 0.0020 & 0.0042 & $<0.0001$ & 0.2308 \\
\hline VLDL (PAG), \% & 0.0024 & 0.0006 & 0.0012 & 0.0036 & 0.0001 & 0.1309 \\
\hline \multicolumn{7}{|l|}{ With statins } \\
\hline $\mathrm{TG}, \mathrm{mmol} / \mathrm{L}$ & 0.0156 & 0.0033 & 0.0089 & 0.0222 & $<0.0001$ & 0.3314 \\
\hline $\mathrm{LDL}-\mathrm{C}, \mathrm{mmol} / \mathrm{L}$ & -0.0077 & 0.0094 & -0.0266 & 0.0113 & 0.4203 & 0.0149 \\
\hline Non - HDL-C, mmol/L & 0.0227 & 0.0072 & 0.0083 & 0.0372 & 0.0027 & 0.1826 \\
\hline TG/LDL-C ratio & 0.0167 & 0.0045 & 0.0077 & 0.0258 & 0.0006 & 0.2349 \\
\hline TG/HDL-C ratio & 0.0113 & 0.0027 & 0.0059 & 0.0167 & 0.0001 & 0.2825 \\
\hline LDL-C/HDL-C ratio & 0.0049 & 0.0085 & -0.0122 & 0.0220 & 0.5687 & 0.0073 \\
\hline Non - HDL-C/HDL-C ratio & 0.0152 & 0.0045 & 0.0061 & 0.0243 & 0.0015 & 0.2018 \\
\hline LDL (PAG), \% & -0.0016 & 0.0006 & -0.0028 & -0.0004 & 0.0110 & 0.1351 \\
\hline Midband (PAG), \% & 0.0033 & 0.0007 & 0.0020 & 0.0046 & $<0.0001$ & 0.3588 \\
\hline VLDL (PAG), \% & 0.0007 & 0.0010 & -0.0013 & 0.0026 & 0.4999 & 0.0102 \\
\hline \multicolumn{7}{|l|}{ Without statins } \\
\hline $\mathrm{TG}, \mathrm{mmol} / \mathrm{L}$ & 0.0199 & 0.0028 & 0.0145 & 0.0254 & $<0.0001$ & 0.4520 \\
\hline $\mathrm{LDL}-\mathrm{C}, \mathrm{mmol} / \mathrm{L}$ & -0.0222 & 0.0076 & -0.0374 & -0.0070 & 0.0050 & 0.1222 \\
\hline Non - HDL-C, mmol/L & 0.0181 & 0.0071 & 0.0038 & 0.0323 & 0.0139 & 0.0953 \\
\hline TG/LDL-C ratio & 0.0240 & 0.0042 & 0.0157 & 0.0323 & $<0.0001$ & 0.3542 \\
\hline TG/HDL-C ratio & 0.0106 & 0.0019 & 0.0068 & 0.0145 & $<0.0001$ & 0.3328 \\
\hline LDL-C/HDL-C ratio & 0.0022 & 0.0088 & -0.0198 & 0.0153 & 0.7994 & 0.0011 \\
\hline Non - HDL-C/HDL-C ratio & 0.0143 & 0.0036 & 0.0071 & 0.0216 & 0.0002 & 0.2033 \\
\hline LDL (PAG), \% & -0.0022 & 0.0005 & -0.0032 & -0.0013 & $<0.0001$ & 0.2668 \\
\hline Midband (PAG), \% & 0.0031 & 0.0008 & 0.0015 & 0.0047 & 0.0003 & 0.1975 \\
\hline VLDL (PAG), \% & 0.0028 & 0.0008 & 0.0012 & 0.0044 & 0.0007 & 0.1713 \\
\hline
\end{tabular}

r: regression coefficient. SE: standard error. $95 \% \mathrm{Cl}$ : 95\% confidence interval

TG triglycerides, LDL-C low-density lipoprotein cholesterol, Non - HDL-C non - high-density lipoprotein cholesterol, HDL-C high-density lipoprotein cholesterol, HDL high-density lipoprotein, $L D L$ low-density lipoprotein, VLDL very low-density lipoprotein, PAG polyacrylamide gel

"Statistical analysis by least squares method

AUC, sensitivity, specificity, and cut-off points for nonHDL-C, TG/LDL-C ratio, and other lipid markers for LDL-MI determination

ROC curves were generated to evaluate the discriminatory ability of 9 lipid markers for LDL-MI, and the AUCs were then calculated (Table 3). Among all patients with $\mathrm{TG} \geq 1.7 \mathrm{mmol} / \mathrm{L}$ analyzed using $\mathrm{LDL}-\mathrm{MI} \geq$
0.400 as the gold standard, the AUC for TG/LDL-C ratio (0.865 [0.786-0.945]) was the highest among the 9 lipid markers examined. The optimal cut-off points for TG/ LDL-C ratio, non-HDL-C, TG/HDL-C ratio, and nonHDL-C/HDL-C ratio for increased LDL-MI were 1.1, $4.9 \mathrm{mmol} / \mathrm{L}, 2.6$, and 3.8, respectively. The sensitivity of the TG/LDL-C ratio for predicting higher sd-LDL was 
Table 3 Comparison of AUC, sensitivity, and specificity for each marker in predicting higher LDL-MI

\begin{tabular}{|c|c|c|c|c|}
\hline Variables & AUC $(95 \% \mathrm{Cl})$ & Sensitivity & Specificity & Cut-off point \\
\hline \multicolumn{5}{|l|}{ Total $(n=110)$} \\
\hline TG & $0.854(0.783-0.926)$ & $79.5 \%$ & $72.7 \%$ & $3.1 \mathrm{mmol} / \mathrm{L}$ \\
\hline$L D L-C^{a}$ & $0.658(0.543-0.773)$ & $61.4 \%$ & $71.2 \%$ & $2.8 \mathrm{mmol} / \mathrm{L}$ \\
\hline Non - HDL-C & $0.643(0.528-0.758)$ & $52.3 \%$ & $81.8 \%$ & $4.9 \mathrm{mmol} / \mathrm{L}$ \\
\hline LDL fraction $(P A G)^{a}$ & $0.766(0.679-0.854)$ & $70.5 \%$ & $66.7 \%$ & $41 \%$ \\
\hline Midband fraction (PAG) & $0.738(0.644-0.832)$ & $75.0 \%$ & $65.2 \%$ & $17 \%$ \\
\hline TG/LDL-C ratio & $0.865(0.786-0.945)$ & $86.4 \%$ & $86.4 \%$ & 1.1 \\
\hline TG/HDL-C ratio & $0.844(0.772-0.917)$ & $75.0 \%$ & $75.8 \%$ & 2.6 \\
\hline LDL-C/HDL-C ratio & $0.516(0.398-0.635)$ & $47.7 \%$ & $62.1 \%$ & 2.2 \\
\hline Non - HDL-C/HDL-C ratio & $0.717(0.615-0.818)$ & $68.2 \%$ & $68.2 \%$ & 3.8 \\
\hline \multicolumn{5}{|l|}{ With statins $(n=47)$} \\
\hline $\mathrm{TG}$ & $0.755(0.604-0.907)$ & $71.4 \%$ & $72.7 \%$ & $3.1 \mathrm{mmol} / \mathrm{L}$ \\
\hline LDL-C $C^{a}$ & $0.466(0.262-0.671)$ & $57.1 \%$ & $57.6 \%$ & $2.8 \mathrm{mmol} / \mathrm{L}$ \\
\hline Non - HDL-C & $0.682(0.500-0.863)$ & $64.3 \%$ & $69.7 \%$ & $4.5 \mathrm{mmol} / \mathrm{L}$ \\
\hline $\mathrm{LDL}$ fraction $(\mathrm{PAG})^{\mathrm{a}}$ & $0.656(0.491-0.821)$ & $64.3 \%$ & $66.7 \%$ & $41 \%$ \\
\hline Midband fraction (PAG) & $0.824(0.695-0.952)$ & $78.6 \%$ & $75.8 \%$ & $21 \%$ \\
\hline TG/LDL-C ratio & $0.697(0.507-0.887)$ & $78.6 \%$ & $78.3 \%$ & 1.1 \\
\hline TG/HDL-C ratio & $0.747(0.602-0.892)$ & $78.6 \%$ & $63.6 \%$ & 2.3 \\
\hline LDL-C/HDL-C ratio & $0.623(0.448-0.799)$ & $64.3 \%$ & $63.6 \%$ & 2.4 \\
\hline Non - HDL-C/HDL-C ratio & $0.691(0.527-0.854)$ & $64.3 \%$ & $69.7 \%$ & 3.5 \\
\hline \multicolumn{5}{|l|}{ Without statins ( $n=63$ ) } \\
\hline TG & $0.910(0.839-0.981)$ & $80.0 \%$ & $87.9 \%$ & $3.3 \mathrm{mmol} / \mathrm{L}$ \\
\hline$L D L-C^{a}$ & $0.760(0.630-0.889)$ & $63.3 \%$ & $90.9 \%$ & $2.6 \mathrm{mmol} / \mathrm{L}$ \\
\hline Non - HDL-C & $0.614(0.463-0.765)$ & $56.7 \%$ & $78.8 \%$ & $5.0 \mathrm{mmol} / \mathrm{L}$ \\
\hline LDL fraction $(P A G)^{a}$ & $0.838(0.735-0.929)$ & $63.3 \%$ & $90.9 \%$ & $35 \%$ \\
\hline Midband fraction (PAG) & $0.701(0.571-0.831)$ & $70.0 \%$ & $69.7 \%$ & $17 \%$ \\
\hline TG/LDL-C ratio & 0.945 (0.884-1.000) & $90.0 \%$ & $93.9 \%$ & 1.1 \\
\hline TG/HDL-C ratio & $0.892(0.815-0.969)$ & $80.0 \%$ & $78.8 \%$ & 2.7 \\
\hline LDL-C/HDL-C ratio & $0.591(0.435-0.747)$ & $53.3 \%$ & $81.8 \%$ & 2.2 \\
\hline Non - HDL-C/HDL-C ratio & $0.724(0.590-0.859)$ & $66.7 \%$ & $81.8 \%$ & 4.3 \\
\hline
\end{tabular}

Cl confidence interval, TG triglycerides, LDL-C low-density lipoprotein cholesterol, Non-HDL-C non-high-density lipoprotein cholesterol, HDL-C high-density lipoprotein cholesterol, PAG polyacrylamide gel

a the lipid markers decrease with the increase of LDL-MI

calculated as $86.4 \%$, with a specificity of $86.4 \%$. Both the sensitivity and specificity of the TG/LDL-C ratio were higher than those of non-HDL-C (52.3 and $81.8 \%$, respectively), TG/HDL-C ratio (75.0 and $75.8 \%$, respectively), and non-HDL-C/HDL-C ratio (68.2 and $68.2 \%$, respectively). Figure $2 \mathrm{~A}$ shows $\mathrm{ROC}$ curves for TG/LDL$\mathrm{C}$ ratio and non-HDL-C among all study patients, and there was significant difference in the AUC between the TG/LDL-C ratio and non-HDL-C $(P=0.0035)$.

Among patients analyzed after grouping by statin use, the AUC for TG/LDL-C ratio (0.945 [0.884-1.000]) in patients not treated with statins was higher than the
AUCs for the other lipid markers in patients with or without statin treatment. Figure 2B shows ROC curves for TG/LDL-C ratio and non-HDL-C in patients not treated with statins, and there was significant difference in the AUC between TG/LDL-C ratio and non-HDL-C $(P=0.0002)$. In patients not treated with statins, the optimal cut-off points for TG/LDL-C ratio, non-HDL-C, TG/HDL-C ratio, and non-HDL-C/HDL-C ratio for increased LDL-MI were $1.1,5.0 \mathrm{mmol} / \mathrm{L}, 2.7$, and 4.3 , respectively. The sensitivity of the TG/LDL-C ratio for predicting higher sd-LDL was calculated as $90.0 \%$, with a specificity of $93.9 \%$. Both the sensitivity and specificity of 


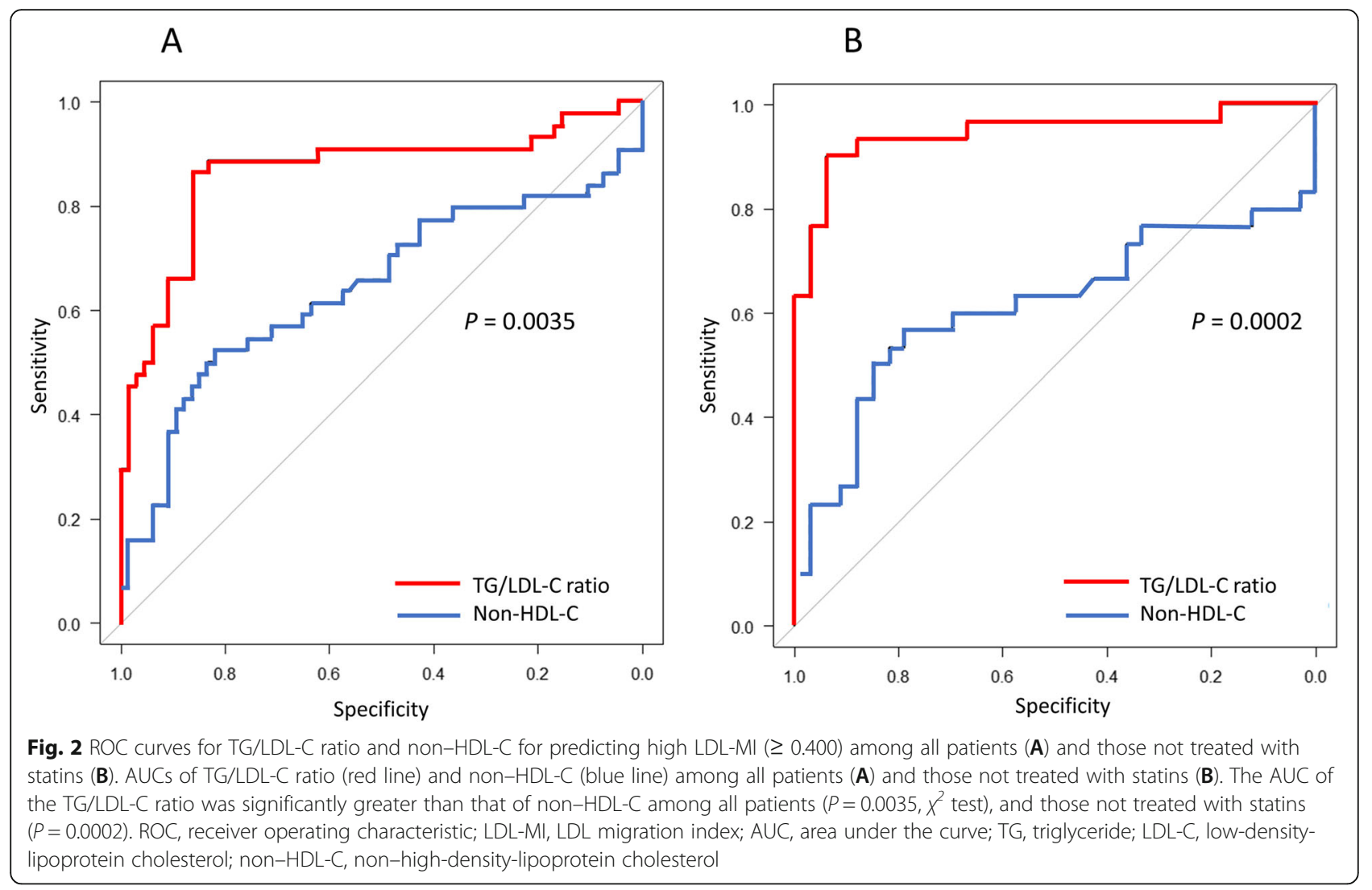

the TG/LDL-C ratio were higher than those of nonHDL-C (56.7 and 78.8\%, respectively), TG/HDL-C ratio (80.0 and $78.8 \%$, respectively), and non-HDL-C/HDL-C ratio (66.7 and $81.8 \%$, respectively). In patients treated with statins, the optimal cut-off points for TG/LDL-C ratio, non-HDL-C, TG/HDL-C ratio, and non-HDL-C/ HDL-C ratio for increased LDL-MI were $1.1,4.5 \mathrm{mmol} /$ L, 2.3, and 3.5, respectively. The sensitivity of TG/LDL$\mathrm{C}$ ratio $(78.6 \%)$ was the same as that of the midband and TG/HDL-C ratio but higher than that of either nonHDL-C (64.3\%) or non-HDL-C/HDL-C ratio (64.3\%). There was no difference in the cut-off point for the TG/ LDL-C ratio for high LDL-MI between patients with and without statin treatment. Although the cut-off points for other lipid markers for higher LDL-MI varied widely depending on statin treatment status, the TG/LDL-C ratio remained constant at approximately 1.1.

\section{Discussion}

The present study demonstrated for the first time the usefulness of the TG/LDL-C ratio as a predictive marker for higher sd-LDL in type 2 diabetes patients with hypertriglyceridemia. In statin-free patients, the assumed cut-off point for TG/LDL-C ratio was 1.1, and the sensitivity and specificity of the ratio as a predictive marker for higher sd-LDL surpassed those of non-HDL-C or other lipid markers. TG/LDL-C ratio is the first formula proposed and considered suitable for evaluation of sdLDL and TG-rich lipoproteins. TGs were positively correlated with LDL-MI, and LDL fraction (PAG) and LDLC were negatively correlated with LDL-MI. The TG/ LDL-C ratio more reliably predicts an increase in LDLMI (sd-LDL). Even if LDL-C is low or within the normal range, it is possible to predict high values of sd-LDL by calculating the relative ratio with TGs. The reciprocal of this formula, LDL-C/TG ratio, was reported by Yoshida et al. and suggested as being related to sd-LDL [18]. In the present study, TG level was used as the numerator for the purpose of emphasizing the existence of TG-rich apoB-containing lipoproteins.

Specific clinical data collected in routine clinical practice can be combined with other related data to increase clinical usefulness for the diagnosis or estimation of various diseases [19]. The routine lipid panel consists of LDL-C, HDL-C, TGs, and total cholesterol. Several additional parameters, such as non-HDL-C (total cholesterol minus HDL-C), LDL-C/HDL-C ratio [20], nonHDL-C/HDL-C ratio [21], and TG/HDL-C ratio [22], are emerging as valuable adjuncts to the standard panel. Non-HDL-C is used to evaluate apoB-containing 
lipoproteins and sd-LDL [23]. In particular, increased non-HDL-C concentration is reportedly associated with residual risk for CVD and has been adopted as a guideline for lipid management [7]. In general, when nonHDL-C increases, the cholesterol contained in TG-rich lipoprotein increases, as do total cholesterol concentrations [24]. In patients with hypertension and/or insulin resistance, the metabolism of lipoproteins is delayed, and they remain in the blood circulation for a variety of reasons [25]. Cholesterol-rich and TG-rich apoBcontaining remnant lipoproteins are taken up by macrophages, and cholesterol accumulates in atherosclerotic lesions [26, 27].

Non-HDL-C is easily calculated by subtracting HDL-C from total cholesterol. Non-HDL-C can provide a better risk estimation compared with LDL-C, in particular in hypertriglyceridemia combined with diabetes, metabolic syndrome, or chronic kidney disease. This is supported by a recent meta-analysis including 14 statin trials, 7 fibrate trials, and 6 nicotinic acid trials [28]. Non-HDL-C is used as an estimation of the total number of atherogenic particles in plasma (VLDL + intermediate-density lipoprotein + LDL) and relates well to apoB levels. Non-HDL-C may be greatly affected by LDL-C and apoB concentrations [29]. A high correlation exists between the changes in non-HDL-C and TGs [30].

In a previous study, it was found that type 2 diabetes patients with hypertriglyceridemia can be divided into two groups: those with relatively low LDL-C and those with normal or high LDL-C. In the former group, TGs and sd-LDL were also higher than those in the latter group, but there was no difference in non-HDL-C between the two groups $(4.9$ [4.0-5.4] $\mathrm{mmol} / \mathrm{L}$ vs. 4.8 [4.55.2] $\mathrm{mmol} / \mathrm{L}$ ) [9]. Alternatively, the increase in TGs and decrease in LDL-C could have been synchronized [13, 31 , and the change in non-HDL-C might have been offset. The non-HDL-C measurement formula evaluates the cholesterol levels of TGs and LDL, which are rich in apoB. An increase in sd-LDL reflecting hypertriglyceridemia and a decrease in large, buoyant LDL might occur simultaneously in type 2 diabetes patients. Patients with type 2 diabetes and/or insulin resistance have increased production of VLDL1, a larger-sized VLDL [32, 33]. VLDL1, after the action of cholesteryl ester transfer protein, produces cholesterol-poor LDL particles (sdLDL) via hydrolysis of TGs by hepatic TG lipase. Simultaneously, the normal size of VLDL is reduced and the large LDL particles produced by the VLDL metabolic process are reduced [33]. As evidence, this study showed that both the LDL-C and LDL fractions in PAG electrophoresis are negatively correlated with LDL-MI. If so, calculated non-HDL-C, regardless of the LDL-C assay method used, will largely reflect the decline in buoyant
LDL present in some type 2 diabetes patients, and it is estimated that the increase in non-HDL-C would not be as expected.

The changes in LDL composition would affect the measurement of non-HDL-C and thus impair its clinical reliability, and these changes might also affect the relationship between non-HDL-C and sd-LDL [9]. In contrast, another lipid marker that indirectly compares the changes in sd-LDL and buoyant LDL (i.e., the TG/LDL$\mathrm{C}$ ratio) seems to more accurately reflect the pathophysiology of dyslipidemia in type 2 diabetes patients. A new subset of atherogenic lipoproteins consisting of LDL-C and TGs is proposed, with LDL-C and TGs serving as surrogates for LDL/intermediate-density lipoprotein and VLDL, respectively. The TG/LDL-C ratio reflects LDL and VLDL and can be a new predictive marker for sdLDL increase. The fact that the cut-off point for the TG/ LDL-C ratio was constant irrespective of statin treatment suggests that the TG/LDL-C ratio is universal and reliable for the prediction of sd-LDL increase. Figure 3 visually summarizes the findings of the present study.

The REDUCE-IT trial showed that treatment with icosapent ethyl significantly reduced CVD events without any change in non-HDL-C in patients with CVD risk and increased baseline TGs but well-controlled LDL-C [30, 34]. The REDUCE-IT trial results may alter the approach to the management of hypertriglyceridemic patients whose lipid phenotype requires more intensive treatment beyond LDL-C lowering alone.

\section{Comparisons with other studies and what the current work adds to existing knowledge}

Yoshida et al. first proposed the LDL-C/TG ratio and total cholesterol (TC)/TG ratio and reported that these ratios were useful as predictors of increased sd-LDL (substituted by LDL-MI) [18]. There are only two reports of formulas for lipid assessment using TG and LDL-C, including the present report. Although patients with normolipidemia were included and no information about glucose tolerance was available, it was reported that the sensitivity and specificity of the TC/TG ratio for predicting high sd-LDL were 72.7 and $83.6 \%$ [18], respectively, values lower than those in present study. In a study involving 994 non-diabetic patients with $\mathrm{TG} \leq 4.5 \mathrm{mmol} / \mathrm{L}(400 \mathrm{mg} / \mathrm{dL})$, the AUC of non-HDL$\mathrm{C}$ was 0.871 (0.840-0.901), and the sensitivity, specificity and positive predictive value for predicting high sd-LDL ( $\geq 46 \mathrm{mg} / \mathrm{dL}$ via direct assay) were 78.8 , 79.8 and $54.9 \%$, respectively [35]. In that study, LDL-C was calculated using Friedewald's formula. A simple comparison between the previous and present studies reveals that the TG/LDL-C ratio of the present study is superior in terms of AUC, sensitivity, and specificity versus non-HDL-C of the previous study for the 


\section{Insulin resistance}

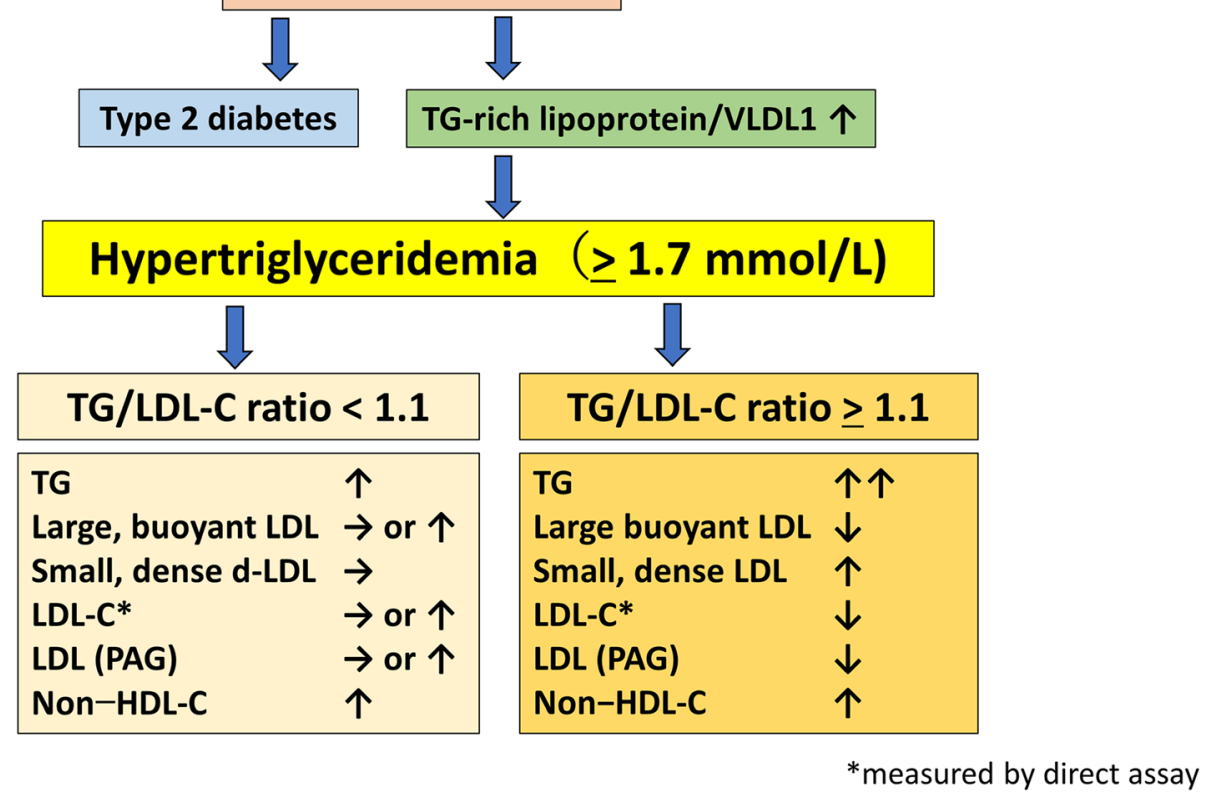

Fig. 3 Graphical summary of the main findings of the present study. TG, triglyceride; LDL-C, low-density-lipoprotein cholesterol; non-HDL-C, nonhigh-density-lipoprotein cholesterol; PAG, polyacrylamide gel electrophoresis

prediction of high sd-LDL. If the usefulness of the TG/ LDL-C ratio in predicting CVD is widely recognized, the recommendations of the atherosclerosis guidelines may need to be revised [7].

\section{Study strengths and limitations}

There are several strengths to this study. First, a reliable LDL-C direct assay, Metabolead LDL-C ${ }^{\bullet}$ (Hitachi Kasei Diagnostic Systems), was used for LDL-C estimation, and the results were consistent with the lipoprotein PAG electrophoresis results [9]. Moreover, this direct method has already been shown to be consistent with ultracentrifugation, unless TGs exceed $11.3 \mathrm{mmol} / \mathrm{L}$ [15, 16]. There were no patients with a fasting TG level of $\geq 11.3 \mathrm{mmol} / \mathrm{L}$ in this study; therefore, any effect of hypertriglyceridemia on the LDL-C assay could be ruled out [36, 37]. A study observing changes in LDL (measured by high-performance liquid chromatography) before and after pemafibrate reported an increase in LDL, especially large, buoyant LDL, in addition to a decrease in sd-LDL after the treatment [38]. Conversely, it indicated that there was a relative reduction in baseline large, buoyant LDL in some patients with hypertriglyceridemia [13]. This is consistent with the decreases in baseline LDL-C (measured via the direct method) and baseline LDL fraction in PAG electrophoresis. Second, PAG electrophoresis, which is a simple and inexpensive method, was used for the estimation of sd-LDL and lipoprotein fractions.

This study also has several limitations. First, instead of directly measuring sd-LDL, determination of LDL-MI by PAG electrophoresis was used as a substitute. However, many reports have indicated that the results of both are strongly correlated [13, 14]. Second, the study design was retrospective; therefore, the reliability of the findings could be inferior comparing with studies of differing design. Third, there were relatively few target patients in this study. Statistically significant results were obtained despite the small number of patients in present study, however. Future studies should enroll a larger number of target patients. Fourth, the prevalence of CVD in the target patients in the present study was low, and it was not possible to evaluate CVD due to quantitative changes in the TG/LDL-C ratio or LDL-MI. Fifth, there may be some specificity in the clinical background of the patients included in present study. There was a high proportion of male patients. Patients treated with statins were older and had lower eGFR. It is generally known that Okinawan subjects (who were analyzed in the present study) tend to develop obesity from an early age and that the frequencies of dyslipidemia and metabolic syndrome in these subjects are high $[39,40]$. It is therefore unclear whether the results of this study are universally applicable to all Japanese or other ethnic groups. 
Finally, this study did not show results for serum apolipoproteins such as apoB, apolipoprotein $\mathrm{E}$ and lipoprotein(a) $[\mathrm{Lp}(\mathrm{a})] . \mathrm{Lp}(\mathrm{a})$ is considered a genetic risk factor for CVD, especially in young people [41]. However, the patients in this study were older and the involvement of $\mathrm{Lp}(\mathrm{a})$. in CVD was presumed to be low.

\section{Conclusion}

In the ACCORD lipid study, combination therapy with a statin and fibrate did not demonstrably suppress CVD events in patients with type 2 diabetes [42]. However, in diabetes patients with high TGs and low HDL-C, the onset of CVD was persistently suppressed even after the ACCORD trial was completed [43]. The TG/LDL-C ratio may offer a simple clinical tool for predicting increased sd-LDL. The TG/LDL-C ratio was useful in the pathologic evaluation of type 2 diabetes patients with high TGs compared with the recommended measurement of non-HDL-C. Even in statin-treated type 2 diabetes patients whose LDL-C remains within the therapeutic range, clear increases in TG/LDL-C ratio and sd-LDL with hypertriglyceridemia are inherent, and pemafibrate or ezetimibe, which have a sd-LDL-lowering effect, might be considered as drugs for add-on therapy $[9,44]$. In addition, statin dosage adjustment may be necessary because of the expected increase in LDL-C associated with the increase in large, buoyant LDL after treatment with these drugs. Assessment of the patient's condition using the TG/LDL-C ratio and appropriate treatment selection may reduce the residual risk of CVD. Further review of the diagnostic indices and control standards for lipids in patients with type 2 diabetes is warranted. A new large-scale clinical trial currently underway is evaluating the usefulness of TG-lowering therapy for suppressing the development of CVD [45]. The usefulness of the TG/LDL-C ratio for CVD prediction may also be demonstrated in that trial.

\section{Acknowledgements}

We would like to thank Dr. Akira Yamamoto (Medical Plaza Daido Central) for collecting patient data and FORTE Science Communications (www.fortescience.co.jp) for English language editing.

\section{Authors' contributions}

IK has full access to all of the data from the study and takes responsibility for the integrity of the data and the accuracy of the data analysis. GO and IK were involved in study design, interpreting the data, statistical analyses, creating tables and figures, and drafting the manuscript. ST was involved in study design. TW and $\mathrm{YO}$ were involved in interpreting the data and supervising the work. All authors have contributed significantly. All the authors have read the manuscript and approved this submission.

\section{Funding}

This research did not receive any specific grant from funding agencies in the public, commercial, or not-for-profit sectors.

\section{Availability of data and materials}

The data that support the findings in this study are available from Medical Plaza Daido Central. Data are available from the authors upon reasonable request and with permission of Medical Plaza Daido Central.

\section{Declarations}

Ethics approval and consent to participate

In accordance with the Declaration of Helsinki, this retrospective study was approved by the Ethics Committee of Medical Plaza Daido Central (approval number 34-2020). Because only medical records were reviewed, patients enrolled in this study were exempted from signing the informed consent.

\section{Consent for publication}

Regarding publication of the research results, we explained that we could not reveal personal information and obtained verbal consent from all target patients.

\section{Competing interests}

The authors declare no conflict of interest.

\section{Author details}

${ }^{1}$ Department of Emergency and Critical Care Medicine, University of the Ryukyus Hospital, 207 Uehara, Nishihara, Okinawa 903-0215, Japan.

2Department of Internal Medicine, Okinawa Medical Hospital, 2310

Tsuhako-Nishihara, Sashiki, Nanjo, Okinawa 901-1414, Japan. ${ }^{3}$ Department of Diabetes and Endocrinology, Medical Plaza Daido Central, 123 Daido, Naha, Okinawa 902-0066, Japan. ${ }^{4}$ Department of Internal Medicine, Okinawa Rehabilitation Center Hospital, 2-15-1 Hiyane, Awase, Okinawa, Okinawa 904-2173, Japan. ${ }^{5}$ Department of Cardiology, Neurology and Nephrology, University of the Ryukyus Hospital, 207 Uehara, Nishihara, Okinawa 903-0215, Japan.

Received: 2 November 2021 Accepted: 7 December 2021

Published online: 07 January 2022

\section{References}

1. Hoogeveen RC, Gaubatz JW, Sun W, Dodge RC, Crosby JR, Jiang J, et al. Small dense low-density lipoprotein-cholesterol concentrations predict risk for coronary heart disease: the Atherosclerosis Risk In Communities (ARIC) study. Arterioscler Thromb Vasc Biol. 2014;34:1069-77. https://doi.org/10.11 61/ATVBAHA.114.303284

2. Chait A, Brazg RL, Tribble DL, Krauss RM. Susceptibility of small, dense, lowdensity lipoproteins to oxidative modification in subjects with the atherogenic lipoprotein phenotype, pattern B. Am J Med. 1993;94:350-6. https://doi.org/10.1016/0002-9343(93)90144-e.

3. Sakai K, Koba S, Nakamura Y, Yokota Y, Tsunoda F, Shoji M, et al. Small dense low-density lipoprotein cholesterol is a promising biomarker for secondary prevention in older men with stable coronary artery disease. Geriatr Gerontol Int. 2018;18:965-72. https://doi.org/10.1111/ggi.13287.

4. Hayashi T, Koba S, Ito Y, Hirano T. Method for estimating high SdLDL-C by measuring triglyceride and apolipoprotein B levels. Lipids Health Dis. 2017. https://doi.org/10.1186/s12944-017-0417-6.

5. Noda H, Iso H, Irie F, Sairenchi T, Ohtaka E, Ohta H. Association between non-high-density lipoprotein cholesterol concentrations and mortality from coronary heart disease among Japanese men and women: the Ibaraki Prefectural Health Study. J Atheroscler Thromb. 2010;17:30-6. https://doi. org/10.5551/jat.1016

6. Manocha A, Bhargava S, Jain R, Kankra M, Singla P, Chugh P. Non-HDL as a valid surrogate marker of small dense LDL in a young Indian population. Indian J Clin Biochem. 2019;34(3):263-71. https://doi.org/10.1007/s12291-01 8-0758-6.

7. Catapano AL, Graham I, De Backer G, Wiklund O, Chapman MJ, Drexel H, et al. 2016 ESC/EAS guidelines for the management of dyslipidaemias. Eur Heart J. 2016;37:2999-3058. https://doi.org/10.1093/eurheartj/ehw272.

8. Robinson JG, Wang S, Smith BJ, Jacobson TA. Meta-analysis of the relationship between non-high-density lipoprotein cholesterol reduction and coronary heart disease risk. J Am Coll Cardiol. 2009;53:316-22. https:// doi.org/10.1016/j.jacc.2008.10.024

9. Komiya I, Yamamoto A, Sunakawa S, Wakugami T. Pemafibrate decreases triglycerides and small, dense $L D L$, but increases $L D L-C$ depending on 
baseline triglycerides and LDL-C in type 2 diabetes patients with hypertriglyceridemia: an observational study. Lipids Health Dis. 2021;20:17. https://doi.org/10.1186/s12944-021-01434-8 pmid: 33610176.

10. Ishibashi S, Yamashita S, Arai H, Araki E, Yokote K, Suganami H, et al. Effects of K-877, a novel selective PPARa modulator (SPPARMa), in dyslipidaemic patients: a randomized, double blind, active- and placebo-controlled, phase 2 trial. Atherosclerosis. 2016;249:36-43. https://doi.org/10.1016/j.a therosclerosis.2016.02.029.

11. Fruchart JC. Pemafibrate (K-877), a novel selective peroxisome proliferatoractivated receptor alpha modulator for management of atherogenic dyslipidaemia. Cardiovasc Diabetol. 2017. https://doi.org/10.1186/s12933-01 7-0602-y.

12. Miller M, Cannon CP, Murphy SA, Qin J, Ray KK, Braunwald E. Impact of triglyceride levels beyond low-density lipoprotein cholesterol after acute coronary syndrome in the PROVE IT-TIMI 22 trial. J Am Coll Cardiol. 2008;51: 724-30. https://doi.org/10.1016/j.jacc.2007.10.038.

13. Tani S, Matsumoto M, Nagao K, Hirayama A. Association of triglyceride-rich lipoproteins-related markers and low-density lipoprotein heterogeneity with cardiovascular risk: effectiveness of polyacrylamide-gel electrophoresis as a method of determining low-density lipoprotein particle size. J Cardiol. 2014; 63:60-8. https://doi.org/10.1016/j.jjcc.2013.06.018.

14. Imajo K, Hyogo H, Yoneda M, Honda Y, Kessoku T, Tomeno W, et al. LDLmigration index (LDL-MI), an indicator of small dense low-density lipoprotein (sdLDL), is higher in non-alcoholic steatohepatitis than in nonalcoholic fatty liver: a multicenter cross-sectional study. PLoS One. 2014. https://doi.org/10.1371/journal.pone.0115403.

15. Miida T, Nishimura K, Hirayama S, Miyamoto Y, Nakamura M, Masuda D, et al. Homogeneous assays for LDL-C and HDL-C are reliable in both the postprandial and fasting state. J Atheroscler Thromb. 2017;24:583-99. https://doi.org/10.5551/jat.40006.

16. Miller WG, Myers GL, Sakurabayashi I, Bachmann LM, Caudill SP, Dziekonski A, et al. Seven direct methods for measuring HDL and LDL cholesterol compared with ultracentrifugation reference measurement procedures. Clin Chem. 2010;56(6):977-86. https://doi.org/10.1373/ clinchem.2009.142810.

17. Kanda Y. Investigation of the freely available easy-to-use software 'EZR' for medical statistics. Bone Marrow Transplant. 2013;48:452-8. https://doi.org/1 0.1038/bmt.2012.244

18. Yoshida A, Kouwaki M, Matsutani Y, Fukuchi Y, Naito M. Usefulness of serum total cholesterol/triglyceride ratio for predicting the presence of small, dense LDL. J Atheroscler Thromb. 2004;11:215-9. https://doi.org/10.5551/ja t.11.215.

19. Hiramatsu K, Yamada T, Yukimura Y, Komiya I, Ichikawa K, Ishihara M, et al. A screening test to identify aldosterone-producing adenoma by measuring plasma renin activity. Results in hypertensive patients. Arch Intern Med. 1981;141(12):1589-93.

20. Kastelein JJP, van der Steeg WA, Holme I, Gaffney M, Cater NB, Barteret P, et al. Lipids, apolipoproteins, and their ratios in relation to cardiovascular events with statin treatment. Circulation. 2008;117:3002-9. https://doi.org/1 0.1161/CIRCULATIONAHA.107.713438.

21. You J, Wang Z, Lu G, Chen Z. Association between the non-high-density lipoprotein cholesterol to high-density lipoprotein cholesterol ratio and the risk of coronary artery disease. Biomed Res Int. 2020;2020:7146028. https:// doi.org/10.1155/2020/7146028.

22. McLaughlin T, Abbasi F, Cheal K, Chu J, Lamendola C, Reaven G. Use of metabolic markers to identify overweight individuals who are insulin resistant. Ann Intern Med. 2003;139:802-9. https://doi.org/10.7326/0003-481 9-139-10-200311180-00007.

23. Pischon T, Girman CJ, Sacks FM, Rifai N, Stampfer MJ, Rimm EB. Non-highdensity lipoprotein cholesterol and apolipoprotein B in the prediction of coronary heart disease in men. Circulation. 2005;112:3375-83. https://doi. org/10.1161/CIRCULATIONAHA.104.532499.

24. Chapman MJ, Ginsberg HN, Amarenco P, Andreotti F, Borén J, Catapano AL, et al. Triglyceride-rich lipoproteins and high-density lipoprotein cholesterol in patients at high risk of cardiovascular disease: evidence and guidance for management. Eur Heart J. 2011;32:1345-61. https://doi.org/10.1093/eurhea rtj/ehr112.

25. Sørensen LP, Andersen IR, Søndergaard E, Gormsen LC, Schmitz O, Christiansen JS, et al. Basal and insulin mediated VLDL-triglyceride kinetics in type 2 diabetic men. Diabetes. 2011;60:88-96. https://doi.org/10.2337/db100564.
26. Joshi PH, Khokhar AA, Massaro JM, Lirette ST, Griswold ME, Martin SS, et al. Remnant lipoprotein cholesterol and incident coronary heart disease: the Jackson Heart and Framingham Offspring Cohort Studies. J Am Heart Assoc. 2016. https://doi.org/10.1161/JAHA.115.002765.

27. Borén J, Williams KJ. The central role of arterial retention of cholesterol-rich apolipoprotein-B-containing lipoproteins in the pathogenesis of atherosclerosis: a triumph of simplicity. Curr Opin Lipidol. 2016;27:473-83. https://doi.org/10.1097/MOL.0000000000000330.

28. Silverman MG, Ference BA, Im K, Wiviott SD, Giugliano RP, Grundy SM, et al. Association between lowering LDL-C and cardiovascular risk reduction among different therapeutic interventions: a systematic review and meta-analysis. JAMA. 2016;316:1289-97. https://doi.org/10.1 001/jama.2016.13985.

29. Ference BA, Kastelein JJP, Ray KK, Ginsberg HN, Chapman MJ, Packard CJ, et al. Association of triglyceride-lowering LPL variants and LDL-C-lowering LDLR variants with risk of coronary heart disease. JAMA. 2019;321:364-73. https://doi.org/10.1001/jama.2018.20045.

30. Marston NA, Giugliano RP, Im K, Silverman MG, O'Donoghue ML, Wiviott SD, et al. Association between triglyceride lowering and reduction of cardiovascular risk across multiple lipid-lowering therapeutic classes: a systematic review and meta-regression analysis of randomized controlled trials. Circulation. 2019;140:1308-17. https://doi.org/10.1161/CIRCULATIONA HA.119.041998.

31. Hirose T, Teramoto T, Abe K, Taneyama T. J-BENEFIT study group. Determinants of bezafibrate-induced improvements in LDL cholesterol in dyslipidemic patients with diabetes. J Atheroscler Thromb. 2015;22:676-84. https://doi.org/10.5551/jat.27425.

32. Hirano H. Pathophysiology of diabetic dyslipidemia. J Atheroscler Thromb. 2018;25:771-82. https://doi.org/10.5551/jat.RV17023.

33. Georgieva AM, van Greevenbroek MMJ, Krauss RM, Brouwers MCGJ, Vermeulen VMM-J, Robertus-Teunissen MG, et al. Subclasses of low-density lipoprotein and very low-density lipoprotein in familial combined hyperlipidemia: relationship to multiple lipoprotein phenotype. Thromb Vasc Biol. 2004;24:744-9. https://doi.org/10.1161/01.ATV.0000119681.4721 8.a4Arteriosclerosis.

34. Boden WE, Bhatt DL, Toth PP, Ray KK, Chapman MJ, Lüscher TF. Profound reductions in first and total cardiovascular events with icosapent ethyl in the REDUCE-IT trial: why these results usher in a new era in dyslipidaemia therapeutics. Eur Heart J. 2020;41:2304-12. https://doi.org/10.1093/eurheartj/ ehz778.

35. Moriyama K, Takahashi E. Non-HDL cholesterol is a more superior predictor of small-dense LDL cholesterol than LDL cholesterol in Japanese subjects with TG levels $<400 \mathrm{mg} / \mathrm{dL}$. J Atheroscler Thromb. 2016;23:1126-37. https:// doi.org/10.5551/jat.33985.

36. Langlois MR, Descamps OS, van der Laarse A, Weykamp C, Baum H, Pulkki K, et al. Clinical impact of direct HDLC and LDLC method bias in hypertriglyceridemia. A simulation study of the EAS-EFLM Collaborative Project Group. Atherosclerosis. 2014;233:83-90. https://doi.org/10.1016/j.a therosclerosis.2013.12.016.

37. Miida T, Nishimura K, Okamura T, Hirayama S, Ohmura H, Yoshida H, et al. A multicenter study on the precision and accuracy of homogeneous assays for LDL-cholesterol: comparison with a beta-quantification method using fresh serum obtained from non-diseased and diseased subjects. Atherosclerosis. 2012;225:208-15. https://doi.org/10.1016/j.atherosclerosis.2 012.08.022.

38. Yamashita S, Arai H, Yokote K, Araki E, Matsushita M, Nojima T, et al. Efficacy and safety of pemafibrate, a novel selective peroxisome proliferatoractivated receptor a modulator (SPPARMa): pooled analysis of phase 2 and 3 studies in dyslipidemic patients with or without statin combination. Int J Mol Sci. 2019;20:5537. https://doi.org/10.3390/ijms20225537.

39. Asato Y, Katsuren K, Ohshiro T, Kikawa K, Shimabukuro T, Ohta T. Relationship between lipid abnormalities and insulin resistance in Japanese school children. Arterioscler Thromb Vasc Biol. 2006;26:2781-6. https://doi. org/10.1161/01.ATV.0000245804.56871.31

40. Mimura G, Nakamasu J, Irie M. Incidence of hyperlipemia in diabetics in Okinawa and its relation to ischemic heart disease. Tohoku J Exp Med. 1983: 141 (Suppl):611-7. https://doi.org/10.1620/tjem.141.suppl_611.

41. Cesaro A, Schiavo A, Moscarella E, Coletta S, Conte C, Gragnano F, et al. Lipoprotein(a): a genetic marker for cardiovascular disease and target for emerging therapies. J Cardiovasc Med (Hagerstown). 2021;22:151-61. https://doi.org/10.2459/JCM.0000000000001077. 
42. ACCORD Study Group, Ginsberg HN, Elam MB, Lovato LC, Crouse JR III, Leiter LA, et al. Effects of combination lipid therapy in type 2 diabetes mellitus. N Engl J Med. 2010;362:1563-74. https://doi.org/10.1056/NEJMoa1 001282.

43. Zhu L, Hayen A, Bell KLL. Legacy effect of fibrate add-on therapy in diabetic patients with dyslipidemia: a secondary analysis of the ACCORDION study. Cardiovasc Diabetol. 2020;19:28. https://doi.org/10.1186/s12933-020-01002-x.

44. Sakamoto K, Kawamura M, Watanabe T, Ashidate K, Kohro T, Tanaka A, et al. Effect of ezetimibe add-on therapy over 52 weeks extension analysis of prospective randomized trial (RESEARCH study) in type 2 diabetes subjects. Lipids Health Dis. 2017;16:122. https://doi.org/10.1186/s12944-017-0508-4.

45. Pradhan AD, Paynter NP, Everett BM, Glynn RJ, Amarenco P, Elam M, et al. Rationale and design of the pemafibrate to reduce cardiovascular outcomes by reducing triglycerides in patients with diabetes (PROMINENT) study. Am Heart J. 2018;206:80-93. https://doi.org/10.1016/j.ahj.2018.09.011.

\section{Publisher's Note}

Springer Nature remains neutral with regard to jurisdictional claims in published maps and institutional affiliations.

Ready to submit your research? Choose BMC and benefit from:

- fast, convenient online submission

- thorough peer review by experienced researchers in your field

- rapid publication on acceptance

- support for research data, including large and complex data types

- gold Open Access which fosters wider collaboration and increased citations

- maximum visibility for your research: over $100 \mathrm{M}$ website views per year

At BMC, research is always in progress.

Learn more biomedcentral.com/submissions 